\title{
Implante intencional de filtros de veia cava em ambas as veias ilíacas comuns: relato de caso e revisão da literatura
}

\author{
Intentional placement of vena cava filters in both iliac veins: case report and literature review \\ Daniel Queiroz Neves', Renvik Demauir Cozine Silva², Luis Claudio Rosa Arantes³, Márcio Cerbazzi Tavares Cardoso ${ }^{4}$, \\ Mauro Henrique de Lima ${ }^{4}$, Gustavo Petorossi Solano ${ }^{5}$, Celso Luis Muhlethaler Chouin ${ }^{5}$, Paulo Eduardo Ocke Reis ${ }^{6}$
}

\begin{abstract}
Resumo
Os filtros de veia cava são utilizados para impedir a passagem de êmbolos dos membros inferiores para as artérias pulmonares e, normalmente, são colocadas imediatamente abaixo das veias renais. Em alguns casos, no entanto, existem dificuldades técnicas incomuns que devem ser superadas para tratar adequadamente alguns pacientes. Relatamos o caso de uma paciente em cujas veias ilíacas comuns foram implantados filtros de veia cava devido à baixa implantação das veias renais e da veia cava inferior curta.
\end{abstract}

Palavras-chave: Trombose venosa; veia cava inferior; filtros de veia cava.

\begin{abstract}
Vena cava filters are used to prevent the passage of emboli from the lower limbs to the pulmonary arteries and normally are placed immediately below the renal veins. In some cases however there are unusual technical difficulties that must be overcome to properly treat some patients. We report a case of a patient in whose common iliac veins vena cava filters were deployed, due to the lower implantation of renal veins and a short inferior vena cava.
\end{abstract}

Keywords: Venous thrombosis; vena cava inferior; vena cava filters.

Introdução

No presente relato, descrevemos o implante de filtros em ambas as ilíacas comuns devido à inserção baixa das veias renais em paciente com trombose venosa de repetição por síndrome do anticorpo antifosfolipídico (SAAF) e falha na terapêutica anticoagulante. Essa variante técnica pode ser uma opção segura e eficaz nos casos em que há impossibilidade de obter acesso à veia cava inferior ou impossibilidade de implante de filtro nesse local.

\section{Descrição do caso}

Paciente de 53 anos apresentou, em 2000, quadro de edema da perna esquerda associado à dor na panturrilha $\mathrm{e}$ no tornozelo. Procurou atendimento médico sendo realizado mapeamento dúplex venoso dos membros inferiores; este revelou trombose venosa profunda (TVP) fêmoro-poplítea esquerda. Foi internada para tratamento anticoagulante e recebeu alta hospitalar após 20 dias fazendo uso de Marevan. Desde então fazia uso contínuo dessa medicação e exames de rotina a cada três meses, sendo o International Normalized Ratio (INR) de difícil controle. Mesmo com anticoagulação plena, a paciente apresentou nove episódios de TVP até 2008, com suspeita de tromboembolismo pulmonar. Nesse período foi diagnosticada SAAF (IgG 31,2 e IgM 28,2).

Em julho de 2009, durante consulta no ambulatório de reumatologia do Hospital Universitário Antônio Pedro a paciente apresentava INR de 6,5 e quadro de dor em perna esquerda associada à paresia, cianose e hipotermia do membro. Foi então internada para investigação. Mapeamento dúplex venoso de membros inferiores revelou trombose parcial aguda de veia femoral direita e das veias femorais profundas bilateralmente.

Trabalho realizado no Hospital Universitário Antônio Pedro da Universidade Federal Fluminense (UFF), Niterói (RJ), Brasil.

${ }^{1}$ Residente (R2) do Serviço de Cirurgia Vascular do Hospital Universitário Antônio Pedro da UFF, Niterói (RJ), Brasil.

${ }^{2}$ Interno do Serviço de Cirurgia Vascular do Hospital Universitário Antônio Pedro da UFF, Niterói (RJ), Brasil.

${ }^{3}$ Chefe de Clínicas do Serviço de Cirurgia Vascular do Hospital Universitário Antônio Pedro da UFF, Niterói (RJ), Brasil.

${ }^{4}$ Médico; Pós-graduando do Serviço de Cirurgia Vascular do Hospital Universitário Antônio Pedro da UFF, Niterói (RJ), Brasil.

${ }^{5}$ Cirurgião Vascular do Serviço de Cirurgia Vascular do Hospital Universitário Antônio Pedro da UFF, Niterói (RJ), Brasil.

${ }^{6}$ Chefe do Serviço de Cirurgia Vascular do Hospital Universitário Antônio Pedro da UFF, Niterói (RJ), Brasil.

Não foram declarados conflitos de interesse associados à publicação deste artigo.

Submetido em: 31.03.2010 Aceito em: 24.05.2010

J Vasc Bras. 2010;9(4):266-269. 
A paciente foi transferida para o Serviço de Cirurgia Vascular que indicou colocação de filtro de veia cava inferior por falha na terapêutica anticoagulante. Foram realizados exames pré-operatórios e troca do Varfarina ${ }^{\circledR}$ por Clexane ${ }^{\circledast}$ cinco dias antes da data prevista para implante do filtro com suspensão deste 12 horas antes do procedimento para evitar complicações hemorrágicas.

Foi utilizado o filtro Günter-Tulip ${ }^{\mathrm{TM}}$ de conformação cônica do grupo dos recuperáveis que possui $30 \mathrm{~mm}$ de diâmetro quando totalmente aberto, bainha de introdução de $8,5 \mathrm{Fr}$, do material Conichrome ${ }^{\bowtie}$, uma liga de metais não ferro-magnético, sendo compatível com exame de ressonância magnética nuclear (Figura 1).

Optou-se, inicialmente, pelo implante do filtro por via jugular direita devido ao relato de trombose venosa profunda no sistema ilíaco direito em Doppler de julho de 2009. Essa via parecia mais segura, pois evita a passagem de fio-guia e cateteres em região com possíveis trombos que podem ser deslocados à passagem de tais materiais. Após cavografia, verificou-se o pequeno comprimento da veia cava inferior, estando a inserção das veias renais próximas à bifurcação das veias ilíacas comuns, o que impossibilitou a colocação do filtro na veia cava infrarrenal como preconizado. Optamos, então, pelo implante de dois filtros, um em cada veia ilíaca comum. Pela via jugular implantou-se um filtro em veia ilíaca comum direita, lado este com maior sintomatologia e trombo mais evidente ao Doppler.

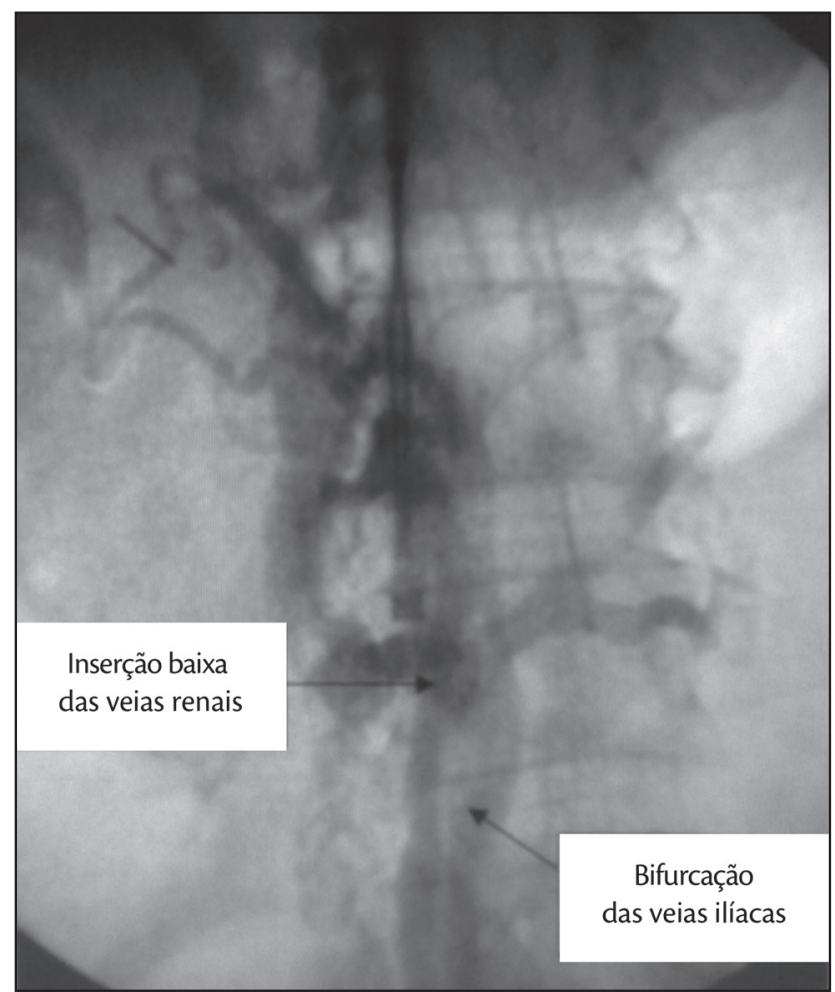

Figura 2 - Implante baixo das veias renais próximo à junção das veias ilíacas comuns
O implante do filtro da veia ilíaca comum esquerda foi realizado por via femoral para evitar o entrelaçamento dos fios-guia e cateteres com o filtro já implantado e seu consequente deslocamento, além do fato de haver maior angulação desse vaso dificultando a colocação do filtro pela via jugular. Tal implante foi também realizado pela técnica de Seldinger, já descrita anteriormente, porém por via femoral esquerda (Figuras 2 e 3 ).

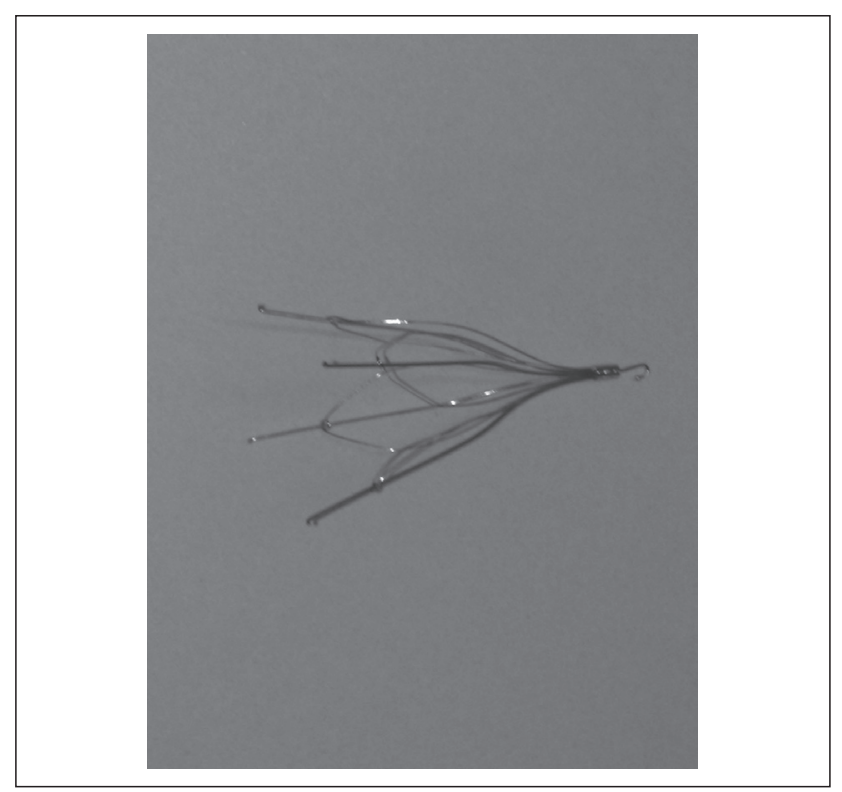

Figura 1 - Filtro utilizado

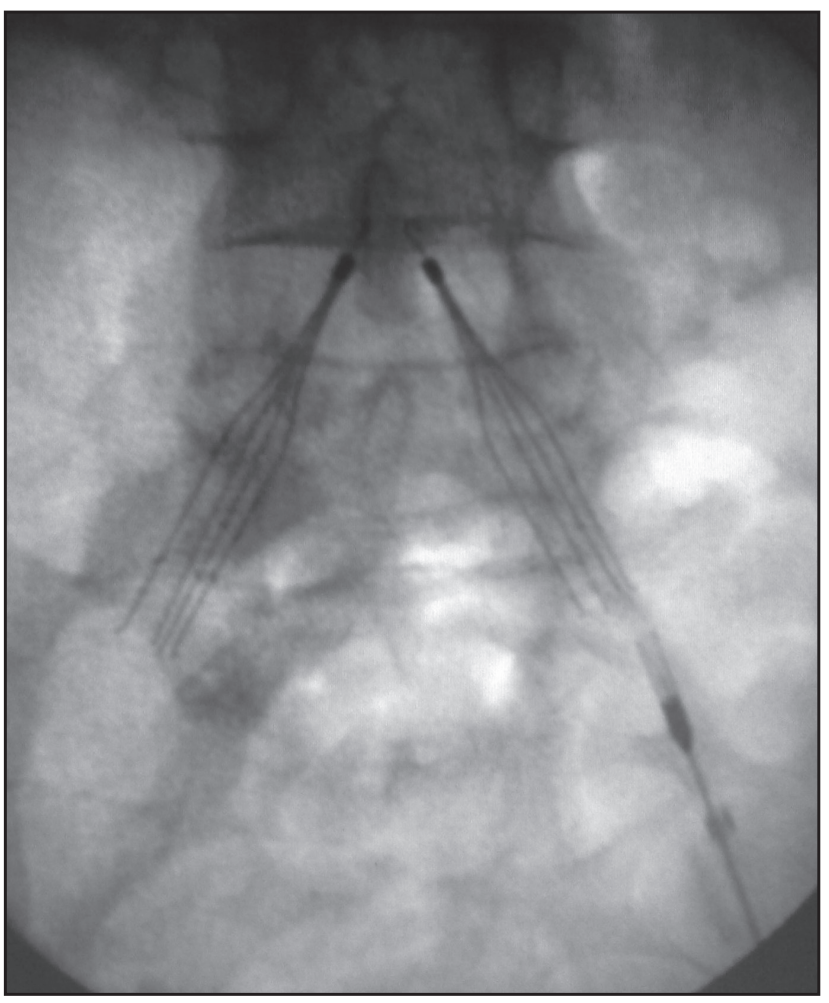

Figura 3 - Filtros em veias ilíacas 
A paciente evoluiu sem intercorrências no pós-operatório imediato sendo reiniciada a anticoagulação com Varfarina ${ }^{\oplus}$. Permaneceu internada por dois dias recebendo alta para acompanhamento ambulatorial. A paciente segue assintomática há seis meses, sem intercorrências inerentes ao procedimento realizado. Um mapeamento dúplex realizado em dezembro de 2009 demonstrou perviedade das veias ilíacas e cava, sem sinais de trombose aguda.

\section{Discussão}

Os filtros de veia cava têm como princípio básico impedir a passagem de êmbolos maiores provenientes dos membros inferiores sem que a veia seja totalmente ocluída, proporcionando uma proteção relativa quanto ao tromboembolismo pulmonar. A porção infrarrenal da veia cava inferior é o local indicado para seu implante, visto que, apesar de ser utilizado para prevenção de eventos embólicos, o filtro não deixa de ser um corpo estranho dentro do vaso e, portanto, exerce algum grau de estímulo trombogênico que pode, em alguns casos, levar a uma trombose sua montante. Dessa forma, seu implante em porção suprarrenal traz o risco de trombose de veia cava com comprometimento das veias renais e suas sérias consequências clínicas ${ }^{1,2}$. Atualmente o filtro de veia cava tem indicações bem definidas, estando indicado em casos de falha da terapia anticoagulante caracterizada por trombose recorrente em vigência de anticoagulação adequada ou quando há contraindicação à terapia anticoagulante ${ }^{3,4}$.

Os filtros são classificados em três categorias ${ }^{3,5}$ : permanentes, temporários e recuperáveis ou opcionais, sendo indicados na prevenção do tromboembolismo pulmonar quando há contraindicação ao uso da terapia anticoagulante, complicações associadas ou falha dessa terapêutica e o seu uso é contraindicado em casos de trombose total da veia cava inferior ou impossibilidade de obter acesso a esse vaso.

Em relação à terapia anticoagulante, 10 a $15 \%$ dos pacientes têm contraindicação a usá-la ou não respondem bem a ela, reembolizando na vigência do tratamento. Zifman et al. ${ }^{6}$ indicaram o implante de filtro de veia cava em 10 pacientes, de um total de 110 indivíduos com SAAF, que continuavam apresentando trombose em vigência de terapia anticoagulante adequada com INR entre 2 e 3 . Em seu estudo, o autor constata que o uso de filtro para profilaxia de tromboembolismo pulmonar é uma conduta eficaz. A paciente do caso descrito encontra-se dentro dessa estatística, pois mesmo em uso de anticoagulação plena continuava apresentando episódios de $\mathrm{TVP}^{7}$ com grande risco de tromboembolismo pulmonar ${ }^{8}$.
Optou-se pelo implante do filtro venoso de conformação cônica do grupo dos recuperáveis ${ }^{9}$, não ferro-magnético, por ser compatível com exames de ressonância nuclear magnética, possuir menor quantidade de metal em sua conformação e menor contato deste com a parede do vaso quando comparados aos de conformação poliédrica e conformação em ninho de pássaro ${ }^{3}$. Isso faz com que seu efeito trombogênico seja atenuado além de causar menor reação endotelial. Apesar de ser do grupo dos opcionais, nesse caso específico ele se tornou permanente devido à impossibilidade de remoção do estímulo trombogênico; nesse caso, a própria $\mathrm{SAAF}^{10-12}$.

Optamos pela manutenção da anticoagulação pósprocedimento, na tentativa de minimizar a recorrência ou exacerbação de TVP, pois o próprio filtro é um agente trombogênico, e a paciente apresenta risco de desenvolver trombos em sítios diversos devido a sua doença de base. Consensos internacionais recomendam anticoagulação perene nos portadores de filtro de veia cava ${ }^{13}$.

Não foram encontrados relatos na literatura associando o implante do filtro bi-ilíaco devido à inserção das veias renais próximas à bifurcação das veias ilíacas comuns e consequente extensão diminuta da veia cava infrarrenal. Os relatos mais frequentes relacionam o implante bi-ilíaco à presença de megacava ${ }^{14,15}$. A inserção baixa das veias renais impossibilitou a colocação do filtro na porção infrarrenal da veia cava inferior bastante diminuída em extensão nessa paciente, fato este que nos levou a optar pelo implante bi-ilíaco em vez da inserção suprarrenal devido ao risco de trombose com envolvimento de veias renais.

O implante de filtro venoso na porção suprarrenal da cava inferior é uma conduta possível, porém há estudos que descrevem casos de trombose de veias renais associado à falência renal em pacientes que se submeteram ao implante de filtro em região suprarrenal ${ }^{1,2}$, relacionando a tríade de Virchow (estase venosa, lesão endotelial e hipercoagulabilidade) à gênese desse processo ${ }^{16}$. O implante suprarrenal do filtro não deixa de ser uma opção; porém, nessa paciente com histórico de nove eventos tromboembólicos em vigência de anticoagulação, nossa equipe julgou mais prudente evitar o envolvimento das veias renais em uma possível trombose de veia cava, optando pela colocação dos filtros nas veias ilíacas. Essa opção terapêutica não minimiza o risco de trombose causado pela implantação dos filtros, mas se ocorrer, provavelmente os danos serão menores do que uma trombose de veia cava com acometimento das veias renais.

A paciente em questão, há nove meses em acompanhamento ambulatorial, segue assintomática. Tal evolução sugere que o implante de filtro em veias ilíacas comuns possa 
ser uma opção segura e efetiva nos casos de veia cava infrarrenal diminuta em extensão e naqueles casos em que o uso de filtro em veia cava inferior está contraindicado.

\section{Conclusão}

Frente ao exposto e conforme a evolução clínica desse caso, foi possível concluir que o filtro é um instrumento terapêutico de grande utilidade quando bem indicado, principalmente em casos como esse, em que a terapia anticoagulante foi ineficaz. Concluiu-se, ainda, que a inserção de filtro biilíaco em substituição ao implante em veia cava é uma opção segura, viável e eficaz em casos selecionados, em que a colocação do filtro em cava infrarrenal não é possível devido a sua curta extensão.

\section{Agradecimentos}

Agradecemos aos doutores Rodrigo Andrade Vaz de Melo, Victor Luiz Picão Correa e ao enfermeiro Sergio Lopes de Azevedo, profissionais que contribuíram na condução desse caso.

\section{Referências}

1. Greenfield LJ, Proctor MC. Suprarenal filter placement. J Vasc Surg 1998;28:432-8.

2. Matchett WJ, Jones MP, McFarland DR, Ferris EJ. Suprarenal vena caval filter placement: follow-up of four filter types in 22 patients. $)$ Vasc Interv Radiol. 1998;9:588-93.

3. Kinney TB. Update on inferior vena cava filters. J Vasc Interv Radiol. 2003; 14:425-40

4. Patel SH, Patel R. Inferior vena cava filters for recurrent thrombosis. Tex Heart Inst I. 2007;34:187-94.

5. Yamagami T, Kato T, lida S, Tanaka O, Nishimura T. Retrievable vena cava filter placement during treatment for deep venous thrombosis. Br J Radiol. 2003;76:712-8.

7. Zifman E, Rotman-Pikielny P, Berlin T, Levy Y. Insertion of inferior vena cava filters in patients with the antiphospholipid syndrome. Semin Arthritis Rheum. 2009;38:472-7.
8. Lensing AW, Prandoni P, Prins MH, Buller HR. Trombose venosa profunda. Lancet. 1999;353:479-85.

9. Kyrle PA, Minar E, Bialonczyk C, Hirschl M, Weltermann A, Eichinger $\mathrm{S}$. The risk of recurrent venous thromboembolism in men and women. N Engl J Med. 2004;350:2558-63.

10. Millward SF, Oliva VL, Bell SD, Valenti DA, Rasul P, Asch M, et al. Günther tulip retrievable vena cava filter: results from the registry of the Canadian Interventional Radiology Association. J Vasc Interv Radiol. 2001;12:1053-8.

11. Louzada Jr. P, Simon SM, Voltarelli JC, Donadi EA. Síndrome do anticorpo antifosfolípide. Medicina, Ribeirão Preto; 1998;31:305-5.

12. Bick RL, Baker WF. O antifosfolipídio e as síndromes trombóticas. Clin Med Am Norte. 1994;3:685-702.

13. Kearon C, Kahn SR, Agnelli G, Goldhaber S, Raskob GE, Comerota AJ . Antithrombotic Therapy for Venous Thromboembolic Disease. Chest. 2008;133:454S-545S.

14. Gomes MP, Kaplan KL, Deitcher SR. Patients with inferior vena caval filters should receive chronic thromboprophylaxis. Med Clin North Am. 2003;87:1189-203.

15. Baron HC, Klapholz A, Nagy AA, Wayne M. Bilateral iliac vein filter deployment in a patient with megacava. Ann Vasc Surg. 1999;13:634-6.

16. Ramchandani P, Zeit RM, Koople HA. Bilateral iliac vein filtration. An effective alternative to caval filtration in patients with megacava. Arch Surg.1991;126:390-3.

17. Marcy PY, Magné N, Frenay M, Bruneton JN. Renal failure secondary to thrombotic complications of suprarenal inferior vena cava filter in cancer patients. Cardiovasc Intervent Radiol. 2001;24:257-9.

Correspondência
Daniel Queiroz Neves
Rua Dr. Celestino, 193 - apto. 202 - Centro
CEP: 24020-090 - Niterói (RJ), Brasil
Telefones: (21) $8787-7810 / 2721-1003$
E-mail: dqneves@yahoo.com.br
Contribuiçães dos autores
Revisão bibliográfica, redação do texto do artigo e acompanhamento clínico da
paciente: DQN
Revisão de prontuário e revisão bibliográfica: RDC
Condução técnica do caso: LCRA
Acompanhamento clínico e ambulatorial da paciente: MCTC, MHL
Acompanhamento e condução clínica da paciente: GPS
Condução técnica do caso: CLMC
Orientação quanto à condução do caso e seu relato: PEOR

